Annuaire du Collège de France 2017-2018

\title{
Théorie économique et organisation sociale (2000-2013)
}

Roger Guesnerie

\section{(2) OpenEdition}

1 Journals

Édition électronique

URL : https://journals.openedition.org/annuaire-cdf/16609

DOI : 10.4000/annuaire-cdf.16609

ISBN : 978-2-7226-0572-5

ISSN : 2109-9227

Éditeur

Collège de France

Édition imprimée

Date de publication : 30 décembre 2020

Pagination : 638-639

ISBN : 978-2-7226-0516-9

ISSN : 0069-5580

\section{Référence électronique}

Roger Guesnerie, «Théorie économique et organisation sociale (2000-2013) », L'annuaire du Collège de France [En ligne], 118 | 2020, mis en ligne le 01 avril 2021, consulté le 22 août 2022. URL : http:// journals.openedition.org/annuaire-cdf/16609; DOI : https://doi.org/10.4000/annuaire-cdf.16609 
THÉORIE ÉCONOMIQUE ET ORGANISATION SOCIALE (2000-2013)

\author{
Roger GUESNERIE
}

Professeur émérite

Mots-clés : économie, crises économiques, changement climatique

\title{
ACTIVITÉS LIÉES À LA RECHERCHE
}

Mon premier et principal sujet de recherche (un sujet sur lequel la crise de 2008, et les défaillances de la prévision qui l'ont suscitée, a renforcé l'actualité d'une réflexion critique), a été, cette année comme les années précédentes, la question de la coordination des anticipations.

Le sujet, pour lequel mon intérêt date du milieu des années 1980, s'inscrit aujourd'hui dans la ligne que portait le réseau mondial de réflexion INEXC (International Network on Expectational Coordination), que j'ai animé jusqu'au début de l'année 2016, et qui comportait, outre Paris, douze pôles de forte visibilité en Europe, Amérique et Asie.

Le cours de 25 heures donné cette année encore dans la formation doctorale M2 de l'École d'économie de Paris, traite aussi de ce sujet, comme l'indique son titre: «Coordination des anticipations : éléments pour une théorie des crises économiques et financières ».

L'essentiel de ma recherche actuelle porte sur les problèmes de coordination dans le contexte macroéconomique. En particulier, le travail engagé depuis un moment avec deux chercheurs d'Outre-Atlantique, qui procède à l'examen d'un des modèles de référence de la macroéconomie, le modèle dit « de cycles réels », a conduit à un texte accepté pour publication (voir ci-dessous).

Mon deuxième sujet de recherche concerne les effets distributifs de la mondialisation. Quels effets aura la poursuite l'intensification du commerce entre le Nord et le Sud sur les inégalités dans les pays développés ? C'est un sujet de débat que le travail engagé depuis quelque temps avec deux autres chercheurs éclaire à partir d'un modèle global stylisé, dont les enseignements sont publiés dans Annals of Economics and Statistics (voir ci-dessous).

Troisième sujet de réflexion: le calcul économique dans l'administration française. Je préside depuis plusieurs années un groupe d'experts sur la mise en œuvre du calcul socio-économique, groupe créé par France Stratégie et le Conseil général pour l'investissement, et ce, en lien avec la direction du Trésor. Un premier document à l'usage de l'administration publique a été publié et diffusé (voir ci-dessous). Ce projet pluriannuel est organisé autour de conférences, réunions, débats, rapports et échanges d'informations.

Quatrième sujet : les questions liées au changement climatique, plus généralement au développement durable, et plus spécifiquement à la valorisation du capital naturel. Ce travail renvoie à l'activité de la chaire "Finance et développement durable » avec laquelle je suis lié. Ce travail s'appuie sur un investissent antérieur 
qui a donné lieu à une série de publications portant à la fois sur la théorie (taux d'actualisation écologique) et la politique économique. L'actualité du sujet continue à susciter des demandes d'interventions diversifiées visant public spécialisé et grand public.

\section{PUBLICATIONS}

GUESNERIE R., CARLUCCIO J. et EKELAND I., «Fragmentation and wage inequality: insights from a simple model », Annals of Economics and Statistics, $\mathrm{n}^{\circ}$ 125-126, Volume in the honour of Edmond Malinvaud, 2017, p. 113-134.

Guesnerie R., Evans G. et Mc Gough B., «Eductive Stability in RBC models », The Economic Journal (sous presse).

Guide de l'évaluation socio-économique des investissements publics, France Stratégie, Direction du Trésor, 2018, rédigé sous l'autorité du comité d'experts des méthodes d'évaluation socioéconomique des investissements publics, sous la présidence de Roger GUESNERIE, professeur au Collège de France. 